\title{
Development of an optical refractometer by analysis of one-dimensional photonic bandgap structures with defects
}

\author{
Ignacio R. Matías, Ignacio Del Villar, and Francisco J. Arregui \\ Departamento de Ingeniería Eléctrica y Eletrónica, Universidad Pública de Navarra, 31006 Pamplona, Spain
}

Richard O. Claus

Fiber and Electro-Optics Research Center, The Bradley Department of Electrical and Computer Engineering, Virginia Polytechnic Institute and State University, 106 Plantation Road, Blacksburg, Virginia 24061

Received January 7, 2003

\begin{abstract}
A theoretical analysis of an optical fiber photonic-bandgap-based refractometer is presented. The design is based on a quarter-wave reflector with one defect. By modifying both the real and the imaginary parts of the index of refraction of the defects we begin to change either the frequency or the amplitude of the localized optical mode. So we could fabricate a specific optical fiber refractometer by combining all the variables: index of refractive index of the defects and the rest of layers, thickness of the defect, number of layers, etc. to yield a large set of possibilities. Some rules for its practical implementation are given. (C) 2003 Optical Society of America
\end{abstract}

OCIS codes: $050.0050,050.2770,060.2310,310.6860$.

Much research has been done with photonic crystals, with many applications in waveguides, ${ }^{1}$ filters, switches, ${ }^{2,3}$ photonic crystal fibers ${ }^{4}$ and sensors, ${ }^{5}$ to mention a few. The reflection output obtained in the photonic bandgaps (PBGs) that originate from these structures is nearly complete, and the introduction of a defect permits localized modes to exist in the range of frequencies of the bandgap. The refractive index and the thickness of the defect define the position of the defect mode in the PBG, making it possible to get out of the structure. ${ }^{6}$ Because of this property, fiber-optic sensors formed by PBG structures with periodicity in one dimension can be fabricated to include a defect layer. This layer is used to detect variations of parameters such as temperature and strain that change the refractive indices of defects. Another important application that we discuss below is the implementation of an optical refractometer by replacement of the defective material. Because the refractive index is the parameter that is measured, the thickness of the defect is the key parameter in terms of design.

The structure of the refractometer is represented in Fig. 1. Two fibers are placed face to face by a precision positioner. The fiber should be single mode to prevent the creation of a set of wave vectors. At the end of each fiber there is a stack of alternately high- and low-refractive-index layers. The depth of the bandgap is defined by the contrast between the refractive indices of the layers and the number of layers of the structure. The defect is represented by a space between the two fibers, where the liquid or gas whose index of refraction is to be detected will be placed. The creation of the defect mode is detected by an optical spectrum analyzer (OSA) at the other side of the fiber. The reason for placing the defect in the middle of the fiber is that the structures at both ends are mirrors that bounce the light of one evanescent mode back and forth. In one dimension this structure could also be understood as constituting distributed Bragg reflectors or even a Fabry-Perot microcavity.

Some experiments that used an electrostatic selfassembly method ${ }^{7-9}$ have successfully investigated the deposition of layers onto the ends of both optical fiber pigtails.

The example presented corresponds to the onedimensional PBG structure shown in Fig. 2, which is exactly a quarter-wave reflector ${ }^{3}$ (QWR) with a defect. It has 61 layers and was designed such that the main bandgap is centered at a standard wavelength of $1550 \mathrm{~nm}$. The electrostatic self-assembly $\operatorname{method}^{7}$ yields two layers with refractive indices $n_{H}=1.8$ [corresponding to $\left(\mathrm{Au}: \mathbf{P D D A}{ }^{+} / \mathbf{P S S}^{-}\right)_{n}$ bilayers] and $n_{L}=1.424$ [corresponding to $\left[\text { poly R-478 } \text { PDDA }^{+} / \mathbf{P s s}^{-}\right]_{n}$ bilayers). ${ }^{8}$ The incident and output media are made from the same fiber. The index of the defect could be that of a the substance, for example, any liquid gas, oil, resin, or blood, to be deposited between the two fibers. Fro example, for Fig. 3 coconut oil, with an index of refraction $1.455,{ }^{10}$ was the fill layer used.

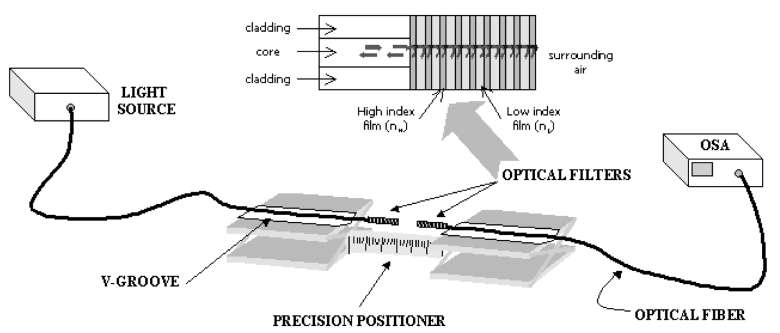

Fig. 1. Structure of the one-dimensional PBG refractometer consisting of two 30-layer Bragg mirrors and a cavity (defect). 


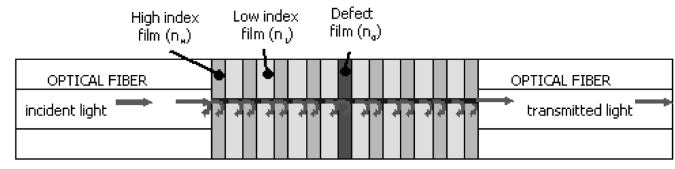

Fig. 2. One-dimensional 61-layer PBG structure with a defect.

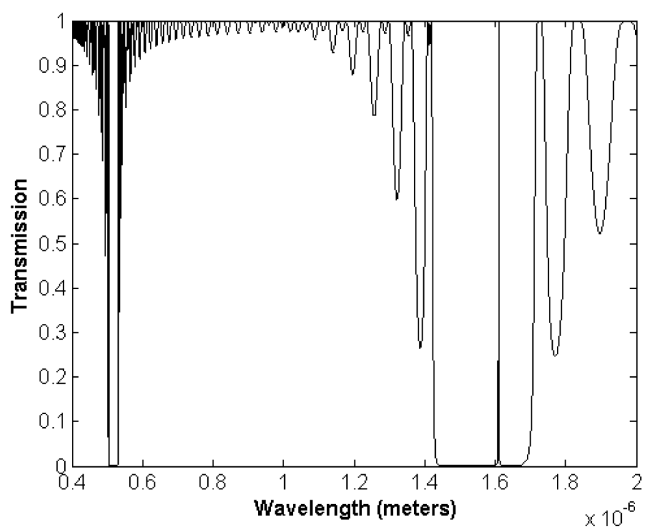

Fig. 3. Transmitted power of the quarter-wave reflector with a defect in the middle.

The thickness of the defect was $100 \mathrm{~nm}$, which avoids the creation of more defects, because they would have thicknesses wider than $200 \mathrm{~nm}$.

The simulation of transmission in this structure is obtained with a program based on rigorous coupled-wave analysis ${ }^{11}$ for a total of 40,000 points in the wavelength range $400-2000 \mathrm{~nm}$. Figure 3 presents two bandgaps, centered at 1550 and $513 \mathrm{~nm}$ and corresponding to first and third harmonics, respectively; the first bandgap is wider. For this reason our analysis is focused on this bandgap. To prove that the structure proposed in this Letter can be used as a refractometer, we show in Fig. 4 transmission plots that correspond to a quarter-wave reflector with a defect in the middle, again $100 \mathrm{~nm}$ thick, but for four refractive indices: $1.455,1.461,1.481$, and 1.518, which correspond to those of coconut oil, tea, cod-liver oil, and tung oil, respectively. ${ }^{10}$ In this case the sweep in wavelength is located in the second bandgap. This design permits the optical spectrum analyzer to distinguish exact wavelength of the localized mode, and it is valuable for a range of refractive indices from 1 to 2, which includes those of all oils of which we know. If the thickness of the defect were reduced, the shift in frequency would be slower; that is the reason for selecting a thickness close to the limit where more localized modes appear. However, it is also possible to increase the accuracy of the device by placing more layers at each side of the defect, because then the peaks would become thinner.

Nevertheless, if a device is desired that detects almost all known refractive indices with the exception of some special polymeric indices, the localized mode must not be shifted out of the photonic bandgap, as it was in the previous design and that one can prevent by reducing the thickness of the defect. This reduction in thickness shifts the peak backward to the middle of the bandgap, at a cost of sensitivity. But the loss can be compensated for by an increase in the number of layers at each side of the defect. In this example a defect is set to $40 \mathrm{~nm}$, and the refractive indices of air, tung oil (1.518), $\mathrm{SiC}$ (2.588), $\mathrm{ZnS}$ (2.881), and GaAs (3.376) are represented in Fig. 5.

As the refractive index of the defect decreases, the central wavelength of the resonator decreases, and the opposite is true for higher refractive indices without resulting in an important change in the location of the PBG. The explanation for this result is that the bandgap is defined by the periodic structure of the photonic crystal, whereas the localized mode is attached to the defect, whose properties (refractive index and thickness) determine its frequency. ${ }^{1}$

The possibility that the material will suffer losses can cause a reduction in the peak of transmission in the structure, something that one can appreciate by examining Fig. 6 for a defect with the same real refractive index of 1.33 (water) and different imaginary parts. There is an additional effect that results from losses. The presence of an imaginary part in the refractive index leads to a reduction of the permittivity of the defect layer. Consequently the frequency of the defect mode is shifted. In Fig. 6 this effect is not obvious because of the small value of the imaginary part of the refractive index. Nevertheless, from Fig. 7 it can be seen that there is a shift between the peak obtained at $1565.42 \mathrm{~nm}$ for a refractive index of $1.33+1 i$

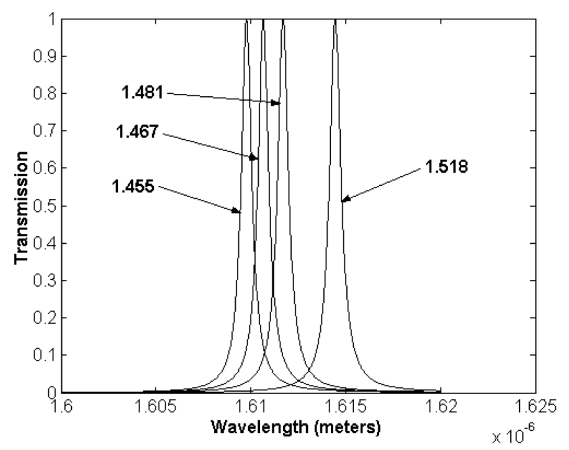

Fig. 4. Transmitted power of a QWR for four refractive indices in the defect. There is no absorption. The thickness of the defect is $100 \mathrm{~nm}$.

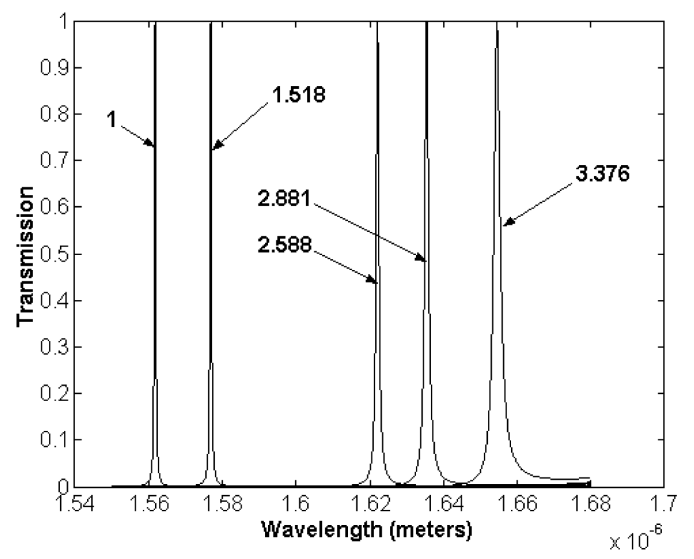

Fig. 5. Transmitted power of a QWR for five refractive indices in the defect. There is no absorption. The thickness of the defect is $40 \mathrm{~nm}$. 


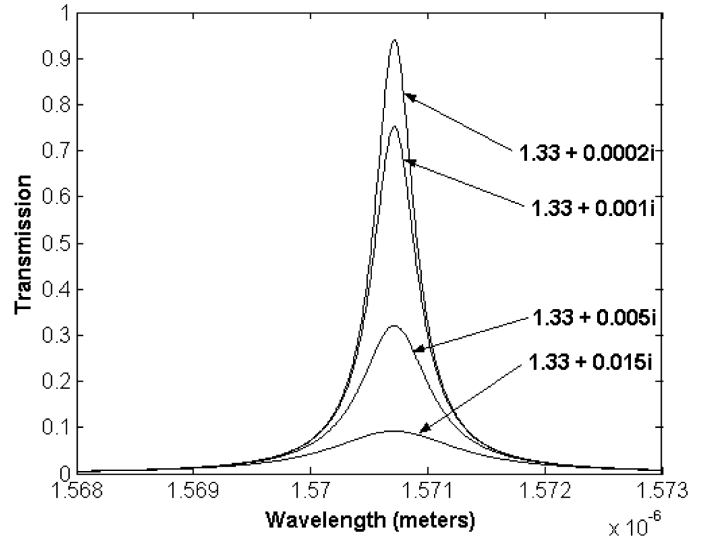

Fig. 6. Transmitted power of a QWR with a defect layer with several imaginary parts in its refractive index. The thickness of the defect is $40 \mathrm{~nm}$.

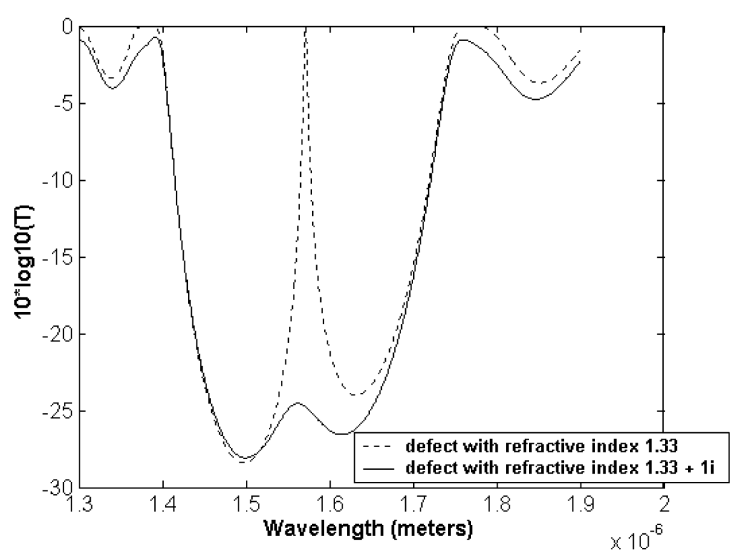

Fig. 7. Transmitted power on a logarithmic scale for a defect with and without losses. The thickness of the defect is $40 \mathrm{~nm}$, and there are 41 layers.

and the peak at 1570.71 for a refractive index of 1.33 . Again this question does not affect the position of the bandgap of the structure (Fig. 7). For the purpose of refractometry it is important to note that the same real refractive index with different losses will lead to different lambdas; consequently for each level of energy transmitted there will be a different range of wavelengths that correspond to concrete refractive indices.

To conclude, it has been shown here that the localized mode in a one-dimensional photonic bandgap structure with a defect can be exploited in the fabrication of an optical refractometer that detects the refractive index of any material. The detection of the power transmitted in the peak of the evanescent mode and its wavelength permits the real refractive index of a material placed in the middle of the device to be detected; it is possible to differentiate between materials with the same real refractive index but different imaginary parts. It is also possible to design wide-range and high-accuracy refractometers by changing some design parameters. The thickness of the defect is the most critical parameter. Small thicknesses of the defect layer in the structure are adequate for detecting wider ranges of refractive indices, and the opposite is true for thin ranges. Besides, the sharpness of the defect mode in the middle of the bandgap can be increased by addition of more layers at both sides of the PBG structure. Finally, the thickness of the defect layer is also limited by the appearance of more than one defect mode at bigger values. This is another application of PBG structures. Following the same rules it could be also applied to two- and three-dimensional structures, but fabrication of these devices is quite complex compared with that of one-dimensional structures, which was our reason for analyzing this type of structure.

This work was supported by grants from the Comisión Interministerial de Ciencia y Tecnología (TIC 2001-0877-C02-02), the Formacion del Profesorado Universitario, Mössbauer Effect Data Center, and the Gobierno de Navarra research (Spain).

\section{References}

1. J. D. Joannopoulos, R. D. Meade, and J. N. Winn, Photonic Crystals: Molding the Flow of Light (Princeton U. Press, Princeton, N.J., 1995).

2. P. R. Villeneuve, D. S. Abrams, S. Fan, and J. D. Joannopoulos, Opt. Lett. 21, 2017 (1996).

3. P. Tran, Opt. Lett. 21, 1138 (1996).

4. J. Broeng, D. Mogilevstev, S. E. Barkou, and A. Bjarklev, Opt. Fiber Technol. 5, 305 (1999).

5. T. D. James, A. C. Greenwald, E. A. Johnson, W. A. Stevenson, J. A. Wollam, T. George, and E. W. Jones, Proc. SPIE Opt. 2000, Photonics West, San Jose, CA, 22-28 January 2000.

6. E. Yablonovitch, J. Opt. Soc. Am. B 10, 283 (1993).

7. F. J. Arregui, I. R. Matías, K. L. Cooper, and R. O. Claus, Opt. Lett. 26, 131 (2001)

8. F. J. Arregui, I. R. Matías, Y. Liu, K. M. Lenahan, and R. O. Claus, Opt. Lett. 24, 596 (1999).

9. F. J. Arregui, B. Dickerson, R. O. Claus, I. R. Matías, and K. L. Cooper, IEEE Photon. Technol. Lett. 13, 1319 (2001)

10. S. A. Khodier, Opt. Laser Technol. 34, 125 (2002).

11. I. R. Matías, I. Del Villar, F. J. Arregui, and R. O. Claus, J. Opt. Soc. Am. A 20, 644 (2003). 